\title{
Author Index Vol. 16, 1996
}

Abbal,M. 478 Abe,S. 309 Abendroth, C.S. 532 Adair,C.D. 529 Adams, R. 402 Adrogué, H.J. 471 Agodoa, L.Y. 7 Ahonen, J. 273 Ahsan,N. 532 Alderman, M.H. 182 Allegra,V. 304 Ammar, K.A. 537 Araki,H. 149 Arif,S. 114 Arora,P. 358 Aunsholt, N.A. 453 Averbukh,Z. 425 Avram, M.M. 118

Baron, E. 478 Barre,P.E. 386 Barroso-Vicens, E. 402 Basçil,N. 367 Bastani, B. 382 Bazzato,G. 489 Bean,J.T. 462 Becker, B.N. 60 Bell,G.M. 106 Ben-Yehuda, A. 520 Bergamo, R. 315 Berland, Y. 159 Berman, S. 425 Bhandari, M. 358 Bignamini, A.A. 489 Biro, A. 162 Bonomini, M. 489 Bonomini, V. 489 Borawski, J. 451 Bottini,P.B. 190 Brancaccio, D. 489 Bremner, F.W. 462 Brezsnyak, W.F. 118 Brod-Miller, C. 280 Broumand, B. 550 Brunswick, J.W. 320 Buckalew,V. 529 Buckalew, V.M., Jr. 138 Bullard,A. 79 Butterly, D.W. 45 Cachera, C. 287, 394 Çaglar, S. 367 Calemard, E. 35 Campese, V.M. 417,446 Cappelli,P. 489 Carr,A.A. 190 Casanova, P. 159 Castelnovo, C. 455 Chan,C.H.S. 144 Chang, D.-M. 293 Charra,B. 35 Chen,C.-H. 300 Chertow, G.M. 79 Chiou,S. 128 Chotigasatit, C. 369 Churg,J. 320 Chyr,S.-H. 293 Cisterne, J.-M. 478 Cohen, L.S. 118 Cohn, M. 425 Corry,D.B. 223 Cossu,M. 489

Dasgupta, A. 327 Davin,J.-C. 500 Dawson, S. 462 Dean,P.B. 506 DeBiase, V. 489 Delmez,J.A. 29 DePaepe,A. 352 Depner, T.A. 17 De Vecchi, A.F. 455 Distler,A. 344 Docci,D. 489 Dracon, M. 287, 394 Dranitzki-Elhalel, M. 520 Durand,D. 478 Dussol, B. 159

Elisaf, M.S. 349 El-Shahawy, M.A. 417,446 El-Sherif,A. 98,114 El-Tantawy, A.E.-H. 114 Epstein, M. 128 Erdem,Y. 367 Ernest, J.M. 529 Evans, C. 529

Fakhry,A. 98 Fanciulli,E. 489 Feigel,D. 162 Feletti,C. 489 Foley,R.N. 386 Fracasso, A. 489 Fricke,H. 484 Friedlaender, M.M. 162

Friedman, E.A. 118 Fruchart, J.-C. 287, 394 Frye,A.H. 529 Fukiyama, K. 375 Fyhrquist, F. 273 Gadallah, M.F. 446 Galmozzi,C. 489 Ghoneim,M. 98, 114 Girone, J.A.C. 553 Goldenberg, R.M. 553 Gomi,H. 167,309 Gower, W.R., Jr. 128 Greco, J. 462 Grosvenor, M. 315 Gulati,S. 358 Gupta, A. 358

Haapanen, A. 506 Hakim, R.M. 60 Harnett,J.D. 386 Hasholt,L. 352 Hayran,M. 367

Haznedaroglu, İ.C. 367 Heckerling, P.S. 537 Held,E. 484 Held,P.J. 7 Hennessy, O. 442 Herman, A.I. 118 Hiraoka, M. 154 Hirschberg, R. 315 Ho,A.S.S. 144 Hochman, B. 162 Hoff,M. 478 Hohenthal,U. 273 Hori,C. 154 Hörl,W.H. 263 Hsiao, P.-H. 300 Hu,L. 386 Huang, Z. 320 Hugue, V. 287 Hurwitz, N. 523

Ifudu,O. 118 Ikeda,K. 309 Iseki,K. 375 Ishimoto,F. 167,309 Ismail, N. 60 Izopet,J. 478 Jacques, M. 327 Jha, R. 358 Jones, C.A. 7

Kanabrocki, E.L. 462 Kandoussi, A.-M. 287 Kandoussi, M. 394 Katzir, Z. 162 Kent,G.M. 386 Kher,V. 358 Kigoshi,T. 91 Kim,S.-T. 412 King,J.W. 446 Kint,J. 352 Kirkland, G.S. 442 Kisugi,R. 309 Kleinert, H.D. 252 Klin,M. 431 Komu,M. 506 Koni,I. 149 Konstantinides, A. 349 Kopple,J.D. 315 Kormano, M. 506 Koss,M. 417 Kovács, T. 500 Krause,P.H. 523 Küchle,C. 484 Kumar, A. 358 Kumar, P. 358 Kun,L. 500 Kuroiwa, A. 412 
Lai,C.KW. 144 Lai,KN. 144 La Manna, G. 489 Lameire, N. 352 Laorpatanaskul, S. 513

Laurent, G. 35 Lazarus, J.M. 79 Lee,J.-S. 300 Leung, C.B. 144 Levin, E.R. 246 Li,B.-L. 293

Li,P.KT. 144 Liaño, F. 268 Lin,Y.-F. 293 Lipschutz, D. 361 Locatelli,F. 489 Lu, K.-C. 293

Mabuchi, H. 149 Maccario, M. 455 McCormick, J.B. 462 McDicken,I. 106 Machida,K 309

McNeely,M. 382 McVeigh, GE. 217 Madsen,K. 352 Maidment, H.J. 52 Malhotra,D. 327

Manning, E.C. 532 Marai,P. 489 Marcén,R. 95,268 Marquet, E. 320

Martimbianco, L. 304 Massry,S.G. 431 Mateos, M.L. 95 Matsuda,H. 167,309 Matsunou, H. 91 Mattana,J. 280 Mayer, G. 263 Mayers, J.D. 118 Mayo,R.R. 361 Mayr,A.C. 523 Mazerska, M. 451 Mengozzi, G. 304 Mesa,C. 417 Messiaen, L. 352 Mezdour,H. 394 Michnowska, M. 431 Mihatsch, MJ. 523 Mioli,V. 489 Misawa, T. 309 Mizukami, Y. 149 Modai, D. 425 Mokrzycki, M.H. 339 Moollaor,P. 513 Moore, L.C. 138 Morimoto, S. 91 Morita,O. 375 Morito,F. 540 Mosconi,G. 489 Moustafa,F. 98 Muramoto, H. 149 Murari, M. 358 Murray, D.C. 386 Mysliwiec, M. 451

Nagy,J. 500 Nakano,H. 309 Nakano,S. 91 Napathorn, S. 513 Nassberger, L. 548 Navarro,J.F. 95,268 Nemchausky, B.M. 462 Neutel,J.M. 202 Niemi,P. 506 Nonomura, A. 149 Norris,S.H. 173

Oda,K. 149 O’Dea,R. 386 Offermann, G. 344 Ohno,I. 309 Ohtsuka,N. 540 Oksman,F. 478 Oldrizzi,L. 489 Ortuño,J. 95,268 Owada,A. 124

Pakkala,S. 273 Pal,S. 280 Pansin,P. 369 Parfrey,P.S. 386

Parra,H.-J. 287 Paul,H. 118 Pawlak,K. 451 Perinbasekar, S. 280 Petersen, J. 52 Piccoli,G. 489 Ponticelli, C. 455 Popovtzer, M.M. 162,520 Posner,L. 339 Pow-Sang,J. 402 Praet,M. 352 Preston, R. 128 Prisant,L.M. 190

Rabb,H. 402 Ramirez, G. 402 Raoult,D. 159 Reade,R. 394 Reade,V. 394 Refaie,A. 114 Ringoir,S. 352 Roccatello, D. 489 Rocco,M.V. 138 Rostaing, L. 478 Rubinger, D.I. 162 Rudwaleit, M. 344 Ryan,M. 462

Saad,M. 114 Safadi,R. 520 Sahai,A. 431 Said,E. 114 Said,R. 334 Sakai,O. 167,309 Sakemi,T. 540 Salmela,K. 273 Savige,J. 442 Sbar, G.D. 320 Scalamogna, A. 455 Scarpioni, L. 489 Scheving, L.E. 462 Schiffl,H. 484 Schmelczer, M. 500 Schmitz,P.G. 382 Schulman, G. 5 Schwab, S.J. 45 Schwarz, A. 344 Shaio,M.-F. 293 Sharma, R, K 358 Sheih,S.-D. 293 Shibasaki,T. 167,309 Shihabi,Z.K. 138 Shiigai,T. 124 Shirazi,P. 462 Siamopoulos, K.C. 349 Silver, J. 162 Sinclair, R.A. 442 Sitprija,V. 369,513 Slomowitz, L.A. 315 Smetana, S. 162

556

Author Index Vol. 16, 1996

SmogOГzewski, M. 431 Sobh,M. 98,114 Sorba,G.B. 489 Sørensen, S.A. 352 Sothern, R.B. 462

Soyannwo, M. 334 Sriboonlue, P. 369 Sridama, V. 513 Steffensen, G. 453 Stefoni, S. 489

Stivelman, J.C. 73 Strøm,E.H. 523 Sudo,M. 154 Suithichaiyakul, T. 513 Suwangool, P. 513

Sverzellati, E. 489

Tacquet,A. 287 Takasugi, M. 412 Tanaka,H. 412 Tankeyoon, M. 513 Taylor, K. 529 Teppo,A.M. 273

Teruel,J.L. 95,268,409 Thamaree, S. 513 Third, J.L.H.C. 462 Tikkanen, I. 273 Todd,J.R. 446

Tomiyoshi,Y. 540 Tosukhowong, P. 369 Trautmann, C. 344 Tsai,W.-S. 300 Tsai,W.-Y. 300

Tsau,Y.-K. 300 Tsuchida, S. 154 Tsugawa, R. 91 Tsukahara,H. 154 Tsushima, Y. 506

Tuck,M.L. 223 Tungsanga, K. 369 Turgan, Ç. 367

Uchida,K. 91 Usui,M. 167 
Vacher-Coponat, H. 159 Vanholder,R. 352 Van Loo, A. 352 Vasile,A. 304 Vecino,A. 409 Veille,J.-C. 529 Venkataseshan, V.S. 320 Vesely,D.L. 128,462

Wagner, L. 500 Weber, M.A. 179,210 Weir,M.R. 237 Weissgarten, J. 425 West,D.C. 106 Wiegand,L $\Lambda .532$ Windus,D.W. 29

Yalçin,A,U. 367 Yaqoob,M. 106 Yasavul,Ü. 367 Yeyati,N.L. 471

Zandinejad, K. 550

AuthorlndexVol. 16,1996 